PROCEEDINGS OF THE

AMERICAN MATHEMATICAL SOCIETY

Volume 141, Number 11, November 2013, Pages 3977-3983

S 0002-9939(2013)11846-0

Article electronically published on July 30, 2013

\title{
SPECTRAL RADIUS OF A NONNEGATIVE MATRIX: FROM ROME TO INDY
}

\author{
MICHAE MISIUREWICZ
}

(Communicated by Nimish Shah)

\begin{abstract}
We generalize the rome method of computing the spectral radius of a nonnegative matrix, used often in one-dimensional dynamics, to the indy method, which works well in many cases when using the rome method is difficult.
\end{abstract}

\section{INTRODUCTION}

There are many situations in which one would like to compute the spectral radius of a nonnegative matrix. This happens for instance in dynamical systems or graph theory. In dynamical systems, topological entropy (and sometimes topological pressure), which is one of the main invariants of a topological dynamical system and tells us how chaotic the system is, can often be computed as a logarithm of the spectral radius of a certain nonegative matrix (see, e.g., [5, 1]). Similarly, in graph theory, the spectral radius of a digraph, defined as the spectral radius of its adjacency matrix (called often in dynamical systems transition matrix), characterizes the complexity of a digraph and is of great importance (see, e.g., 3]).

While for a single matrix $M$ one can use many computer programs to quickly compute its spectral radius $\operatorname{spr}(M)$ (although this was not the case 30 or more years ago), the situation is not that simple. First, one may be more interested in finding a polynomial whose zero is $\operatorname{spr}(M)$ rather than the approximate numerical value of $\operatorname{spr}(M)$. This task can also be performed by a computer, but the form of a polynomial will not necessarily be the simplest one. Second, the dimension of the matrix may be large, and its input into the program may take considerable time. Third, instead of a single matrix, one often considers a family of matrices with some special structure of larger and larger dimensions. Then the desired outcome is some formula depending on the parameters, and for this more than a computer program may be necessary.

In 1980, the rome method was invented in 2] to overcome those problems. It works very well for problems appearing in one-dimensional dynamics (see, e.g., 1]). In the interpretation via a digraph (with edges carrying nonnegative weights), we need to find a small rome, that is, a set of vertices such that all infinite paths pass through it. While in many cases this is possible, sometimes (for instance, if most of the diagonal entries of the matrix are positive) this does not work. Here we present a generalization of this method, which we call the indy method (Indianapolis is not

Received by the editors January 29, 2012.

2010 Mathematics Subject Classification. Primary 37B40, 15A18; Secondary 05C50, 05C20.

Key words and phrases. Spectral radius, digraph, topological entropy, rome method. 
Rome...). While the restrictions on the set of vertices are absent in this method, polynomials appearing in the rome method are replaced by a power series. Checking that those power series converge makes the proof in the indy case considerably more complicated than in the rome case.

The paper is organized as follows. In Section 2 we fix the notation used later. In Section 3 we describe the rome method. In Section 4 we describe the indy method and prove that it works. In Section 5 we present a simple example when the rome method works very well and then modify it so that the rome method does not give any advantage, while the indy method works very well.

\section{NotATion}

We consider square matrices. For two such matrices $M=\left(m_{i j}\right)_{i, j=1}^{d}$ and $N=$ $\left(n_{i j}\right)_{i, j=1}^{d}$ we write $M \leq N$ if $m_{i j} \leq n_{i j}$ for every $i, j$. If $M \leq N$ and $M \neq N$, we will write $M \prec N$ (writing $M<N$ would be more logical, but confusing, since it could be understood as $m_{i j}<n_{i j}$ for every $i, j$ ). The zero matrix of any size will be denoted just 0 , and the unit matrix $I$. Thus, $M \geq 0$ means that the matrix $M$ is nonnegative (has all entries nonnegative).

Let $M=\left(m_{i j}\right)_{i, j=1}^{d}$ be a nonnegative matrix. A sequence $p=\left(i_{0}, i_{1}, \ldots, i_{n}\right)$ of elements of $\{1, \ldots, d\}$ will be called a road from $i_{0}$ to $i_{n}$. Then $n$ will be called the length of $p$ and be denoted by $\ell(p)$, while the product

$$
\prod_{k=1}^{n} m_{i_{k-1} i_{k}}
$$

will be called the width of $p$ and be denoted by $w(p)$. The function $y \mapsto w(p) y^{\ell(p)}$ will be called the characteristic of $p$ and be denoted by $\operatorname{ch}(p)$.

For two roads $p=\left(i_{0}, i_{1}, \ldots, i_{n}\right)$ and $q=\left(j_{0}, j_{1}, \ldots, j_{\nu}\right)$, if $i_{n}=j_{0}$, then we can form their concatenation $p q=\left(i_{0}, i_{1}, \ldots, i_{n}, j_{1}, \ldots, j_{\nu}\right)$. Clearly, $\operatorname{ch}(p q)=$ $\operatorname{ch}(p) \operatorname{ch}(q)$.

A road $p=\left(i_{0}, i_{1}, \ldots, i_{n}\right)$ passes through $j \in\{1, \ldots, d\}$ if there is $k \in\{1, \ldots$, $n-1\}$ such that $i_{k}=j$. For $i, j \in\{1, \ldots, d\}$ and $S \subset\{1, \ldots, d\}$, we denote by $D(i, j, S)$ the set of all roads from $i$ to $j$ not passing through any element of $S \cup\{i, j\}$. We set

$$
f_{i, j, S}=\sum_{p \in D(i, j, S)} \operatorname{ch}(p)
$$

and if $S$ is nonempty, we set

$$
M_{S}=\left(f_{i, j, S}\right)_{i, j \in S}
$$

Finally, we will denote the spectral radius of a matrix $M$ by $\operatorname{spr}(M)$ and its characteristic polynomial by $\chi_{M}$. The determinant of a matrix $M$ will be denoted by $\operatorname{det}(M)$. For a finite set $S$ its cardinality will be denoted by $|S|$.

\section{ROME METHOD}

In dynamical systems topological entropy is often equal to the logarithm of the spectral radius of some nonnegative matrix. If this matrix has many zeros, then in order to compute its spectral radius one can use a very effective (especially for computations "by hand") rome method. It has been introduced in [2]. 
Let $M=\left(m_{i j}\right)_{i, j=1}^{d}$ be a matrix. A subset $R \subset\{1, \ldots, d\}$ is called a rome if all roads lead to it (omnes viae Romam ducunt); that is, for every $i \in\{1, \ldots, d\} \backslash R$ every road of nonzero width from $i$ to $i$ passes through some element of $R$. The following theorem can be found for instance in [2] or [1].

Theorem 3.1. If $R$ is a rome for a $d \times d$ matrix $M$, then

$$
\chi_{M}(x)=(-1)^{d-|R|} x^{d} \operatorname{det}\left(M_{R}\left(x^{-1}\right)-I\right) .
$$

If $M$ is a nonnegative matrix, then its spectral radius is equal to the largest real zero $\chi_{M}$. Therefore we get the following corollary.

Corollary 3.2. If $R$ is a rome for a $d \times d$ nonnegative matrix $M$ with positive spectral radius, then $\operatorname{spr}(M)$ is the reciprocal of the smallest positive zero of the polynomial $\operatorname{det}\left(M_{R}(y)-I\right)$.

\section{INDY METHOD}

We want to generalize the rome method to the case when $R$ is not necessarily a rome. Since unfortunately not all roads lead to Indy (Indianapolis), we can call any subset $R \subset\{1, \ldots, d\}$ an indy. Then the entries of the matrix $M_{R}$ will be a series rather than polynomials. Therefore, in order to recover the characteristic polynomial of $M$, as in (3.1) we would have to multiply $\operatorname{det}\left(M_{R}\left(x^{-1}\right)-I\right)$ by $x$ to the infinite power. This of course does not make sense, so we will generalize Corollary 3.2 rather than Theorem 3.1 .

In order to control the radii of convergence of the series that will appear in the formulas, we will use the following theorem. It is an immediate corollary to a somewhat more general Wielandt's Theorem on spectral radii (see, e.g., 4]).

Theorem 4.1. Let $M$ be a nonnegative irreducible $d \times d$ matrix, and let $N$ be a nonnegative $d \times d$ matrix such that $N \prec M$. Then $\operatorname{spr}(N)<\operatorname{spr}(M)$.

Before we state our main result, we will prove some lemmas that will be used in its proof.

Lemma 4.2. Let $M$ be a nonnegative $d \times d$ matrix with positive spectral radius. Then the radius of convergence of the series

$$
\sum_{p} \operatorname{ch}(p)
$$

where the summation is over all roads $p$, is equal to $1 / \operatorname{spr}(M)$.

Proof. Think of the formula for the $i j$-th entry of the matrix $M^{n}$, where $M=$ $\left(m_{i j}\right)_{i, j=1}^{d}$. It is the sum of all products of the form $m_{i i_{1}} m_{i_{1} i_{2}} \ldots m_{i_{n-2} i_{n-1}} m_{i_{n-1} j}$. That is, it is the sum of widths of all paths of length $n$ from $i$ to $j$. If $M \geq 0$, all numbers involved are nonnegative. The spectral radius of $M$ is equal to the growth rate of the sum of all entries of $M^{n}$, so it is equal to the growth rate of the sum of widths of all paths of length $n$. Now we use the standard formula for the radius of convergence of a power series.

Lemma 4.3. Let $M$ be a nonnegative $d \times d$ matrix with positive spectral radius. If $R=\{1, \ldots, d\}$, then $\operatorname{spr}(M)$ is the reciprocal of the smallest positive zero of $\operatorname{det}\left(M_{R}(y)-I\right)$. 
Proof. For every $i, j$ we have $D(i, j, R)=\{(i, j)\}$, so $f_{i, j, R}(y)=m_{i j} y$. Thus, if $y \neq 0$, we get

$$
\operatorname{det}\left(M_{R}(y)-I\right)=y^{d} \operatorname{det}\left(M-\frac{1}{y} I\right)=y^{d} \chi_{M}\left(\frac{1}{y}\right) .
$$

Since $\operatorname{spr}(M)$ is the largest zero of $\chi_{M}$, we see that it is the reciprocal of the smallest positive zero of $\operatorname{det}\left(M_{R}(y)-I\right)$.

Lemma 4.4. Let $M$ be a nonnegative irreducible $d \times d$ matrix. Let $S$ be a nonempty subset of $\{1, \ldots, d\}$ and let $k \in\{1, \ldots, d\} \backslash S$. Set $R=S \cup\{k\}$. Then

$$
\operatorname{det}\left(M_{R}(y)-I\right)=\operatorname{det}\left(M_{S}(y)-I\right)\left(f_{k, k, R}(y)-1\right)
$$

for all $y>0$ for which the series appearing in (4.2) converge. Moreover, for such $y$ we have $\left|f_{k, k, R}(y)\right|<1$.

Proof. Look at a road $p \in D(i, j, S)$, where $i, j \in S$. If it does not pass through $k$, then it belongs to $D(i, j, R)$. If it passes $n+1 \geq 1$ times through $k$, then it is a concatenation of a road from $D(i, k, R)$, then $n$ roads from $D(k, k, R)$, and finally a road from $D(k, j, R)$. Each such road belongs to $D(i, j, S)$. Thus,

$$
f_{i, j, S}=\sum_{p \in D(i, j, R)} \operatorname{ch}(p)+\sum_{n=0}^{\infty}\left(\sum_{q \in D(i, k, R)} \operatorname{ch}(q) \sum_{r \in D(k, j, R)} \operatorname{ch}(r)\left(\sum_{s \in D(k, k, R)} \operatorname{ch}(s)\right)^{n}\right)
$$

$$
=f_{i, j, R}+f_{i, k, R} \cdot f_{k, j, R} \cdot \sum_{n=0}^{\infty}\left(f_{k, k, R}\right)^{n} .
$$

If $\left|f_{k, k, R}(y)\right|<1$, then we get

$$
f_{i, j, S}=f_{i, j, R}+f_{i, k, R} \cdot f_{k, j, R} \cdot \frac{1}{1-f_{k, k, R}} .
$$

To compute $\operatorname{det}\left(M_{R}-I\right)$, we can add to each column of this matrix indexed by $j \in S$ the column indexed by $k$ multiplied by $f_{k, j, R} /\left(1-f_{k, k, R}\right)$. If we denote the entries that we get this way by $g_{i, j}(i, j \in R)$, then:

$$
g_{k, k}=f_{k, k, R}-1
$$

for $j \neq k$ we have

$$
g_{k, j}=f_{k, j, R}+\left(f_{k, k, R}-1\right) \cdot \frac{f_{k, j, R}}{1-f_{k, k, R}}=0
$$

for $i, j \in S$, if $i \neq j$ we have

$$
g_{i, j}=f_{i, j, R}+f_{i, k, R} \cdot \frac{f_{k, j, R}}{1-f_{k, k, R}} ;
$$

so by (4.4), $g_{i, j}=f_{i, j, S}$ and, similarly, if $i \in S$, then $g_{i, i}=f_{i, i, S}-1$. Thus, (4.2) holds. However, we had to assume that we were working with convergent series and that $\left|f_{k, k, R}(y)\right|<1$. 
Now, remove the assumption that $\left|f_{k, k, R}(y)\right|<1$ and suppose that $\left|f_{k, k, R}(y)\right| \geq$ 1. Since $M$ is irreducible, there are $i, j \in S$ such that the sets $D(i, k, R)$ and $D(k, j, R)$ are nonempty, and therefore $f_{i, k, R}$ and $f_{k, j, R}$ are positive. Then in (4.3) we get as a value at $y$ a finite number on the left-hand side, but infinity on the right-hand side, a contradiction. Therefore we get $\left|f_{k, k, R}(y)\right|<1$ whenever the series we are working with are convergent.

Lemma 4.5. Let $M=\left(m_{i j}\right)_{i, j=1}^{d}$ be a nonnegative irreducible matrix. Then for every $i, j \in\{1, \ldots, d\}$ and $S \subset\{1, \ldots, d\}$ the radius of convergence of the series (2.1) is strictly larger than $1 / \operatorname{spr}(M)$.

Proof. We can write $D(i, j, S)$ as the union of its subsets $D_{k}$ consisting of the roads that begin with $(i, k)$, over all $k \in\{1, \ldots, d\} \backslash\{i\}$ (and the singleton of the path $(i, i)$ if $j=i)$. The characteristic of any road from $D_{k}$ is equal to $m_{i k} y$ times the characteristic of this road without the initial $i$. However, such a road is also a road from $k$ to $j$ for the matrix $N$ which is obtained from $M$ by replacing all entries $m_{i \ell}(\ell=1, \ldots, d)$ by 0 . Thus, by Lemma 4.2. the radius of convergence of the series (2.1) is at least $1 / \operatorname{spr}(N)$.

Clearly, $N \leq M$. Since $M$ is irreducible and $N$ is not, we have $N \neq M$, and thus $N \prec M$. By Theorem 4.1, $\operatorname{spr}(N)<\operatorname{spr}(M)$. Therefore, the radius of convergence of the series (2.1) is strictly larger than $1 / \operatorname{spr}(M)$.

Now we can prove our main theorem.

Theorem 4.6. Let $M$ be a nonnegative $d \times d$ irreducible matrix. Then for any indy $S$ (a nonempty subset of $\{1, \ldots, d\}) \operatorname{spr}(M)$ is the reciprocal of the smallest positive zero of $\operatorname{det}\left(M_{S}(y)-I\right)$.

Proof. Since $M$ is nonnegative and irreducible, it has a positive spectral radius, so we can use Lemma 4.3. Then we use induction, removing one by one the elements of $\{1, \ldots, d\} \backslash S$, and using Lemmas 4.4 and 4.5. At each stage of induction we have an indy $R$, whose smallest positive zero is $1 / \operatorname{spr}(M)$.

\section{EXAMPLE}

Let us illustrate a simple example of how the rome and indy methods can work. Let $M=\left(m_{i j}\right)_{i, j=1}^{n+1}$ be a 0 - 1 matrix with $m_{1 i}=m_{i 1}=1$ for $i=2, \ldots, n+1$ and the rest of the entries 0 . It is the transition (adjacency) matrix of the digraph shown in Figure 1,

We can compute the spectral radius of $M$ immediately by using the rome method with the rome $R=\{1\}$. The set $D(1,1, R)$ consists of $n$ roads of length 2 and width 1 each, so $f_{1,1, R}(y)=n y^{2}$. Thus, by Corollary [3.2 $\operatorname{spr}(M)$ is the reciprocal of the smallest positive solution to the equation $n y^{2}=1$, that is, $\operatorname{spr}(M)=\sqrt{n}$.

Now consider the matrix $N$, obtained from $M$ by replacing the diagonal entries, except the first one, by 1 . It is the transition matrix of the digraph shown in Figure 2. Now using the rome method will not give us any big advantage, because there are only 2 possible romes, $\{1,2, \ldots, n+1\}$ and $\{2, \ldots, n+1\}$, and they are big. However, we can compute the spectral radius of $N$ very easily using the indy 


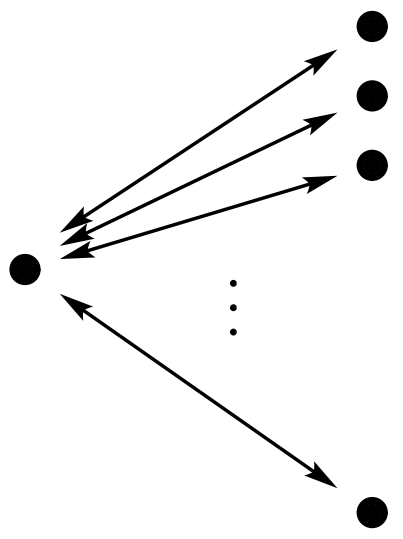

Figure 1. The digraph whose transition matrix is $M$.

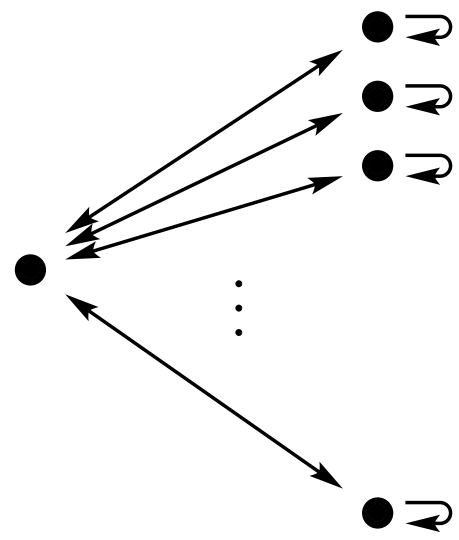

FiguRE 2. The digraph whose transition matrix is $N$.

method with the indy $R=\{1\}$. The set $D(1,1, R)$ now consists of $n$ roads of length $k$ for all $k \geq 2$. All roads have width 1 . Therefore

$$
f_{1,1, R}(y)=n \sum_{k=2}^{\infty} y^{k}=\frac{n y^{2}}{1-y} .
$$

Clearly, the matrix $N$ is irreducible. Thus, by Theorem 4.6, $\operatorname{spr}(M)$ is the reciprocal of the smallest positive solution to the equation

$$
\frac{n y^{2}}{1-y}=1
$$

that is, of the equation $n y^{2}=(1-y)$. Substituting $x=1 / y$, we get the equation $x^{2}-x-n=0$, and thus,

$$
\operatorname{spr}(N)=\frac{1+\sqrt{1+4 n}}{2}
$$




\section{REFERENCES}

[1] Lluís Alsedà, Jaume Llibre, and Michał Misiurewicz, Combinatorial dynamics and entropy in dimension one, 2nd ed., Advanced Series in Nonlinear Dynamics, vol. 5, World Scientific Publishing Co. Inc., River Edge, NJ, 2000. MR1807264 (2001j:37073)

[2] Louis Block, John Guckenheimer, Michał Misiurewicz, and Lai Sang Young, Periodic points and topological entropy of one-dimensional maps, Global theory of dynamical systems (Proc. Internat. Conf., Northwestern Univ., Evanston, Ill., 1979), Lecture Notes in Math., vol. 819, Springer, Berlin, 1980, pp. 18-34. MR591173 (82j:58097)

[3] Richard A. Brualdi, Spectra of digraphs, Linear Algebra Appl. 432 (2010), no. 9, 2181-2213, DOI 10.1016/j.laa.2009.02.033. MR2599853 (2011g:05003)

[4] Carl Meyer, Matrix analysis and applied linear algebra, Society for Industrial and Applied Mathematics (SIAM), Philadelphia, PA, 2000. With 1 CD-ROM (Windows, Macintosh and UNIX) and a solutions manual (iv+171 pp.) MR.1777382

[5] Peter Walters, An introduction to ergodic theory, Graduate Texts in Mathematics, vol. 79, Springer-Verlag, New York, 1982. MR648108 (84e:28017)

Department of Mathematical Sciences, Indiana University-Purdue University Indianapolis, 402 N. BlaCkFord Street, Indianapolis, Indiana 46202

E-mail address: mmisiure@math.iupui.edu 